\title{
Simulation of Distributed Wireless Networked Control Systems over MANET using OPNET
}

\author{
M S Hasan, Hongnian Yu, Alison Griffiths and T C Yang* \\ Faculty of Computing, Engineering and Technology, Staffordshire University, UK \\ *Department of Engineering and Design, University of Sussex, UK \\ \{m.s.hasan, h.yu, a.l.griffiths\}@staffs.ac.uk, *t.c.yang@sussex.ac.uk
}

\begin{abstract}
This paper investigates the simulation of distributed Wireless Networked Control Systems (WNCS) over Mobile Ad-hoc NETwork (MANET). The simulation model includes multiple plants with a single controller and random node mobility. The widely used network simulation software OPtimised Network Engineering Tool (OPNET) has been utilised to implement a realistic wireless signal propagation model using path loss and fading effects. System performances for two ad-hoc network routing protocols: DSR and AODV have been explored.
\end{abstract}

Keywords- Distributed Wireless Networked Control Systems, Mobile Ad-hoc Network, OPNET, Wireless Signal propagation model, path loss exponent, fading effect, DSR routing algorithm, AODV routing algorithm.

\section{INTRODUCTION}

Networked Control Systems (NCS) have been used in industrial applications for several years as they offer many advantages over traditional control systems [1]. Recently attention is being paid to Wireless Networked Control Systems (WNCS) because of high performance portable computers and wireless networks. WNCS can be very useful in ad-hoc applications, for instance, military use, rescue operation, assembling space structures, exploring hazardous environment, executing tele-surgery [2], online aircraft monitoring [3] etc.

Mobile Ad-hoc NETworks (MANET) offer very dynamic and flexible wireless networks; these are self-organising and can be easily deployed without any infrastructure [4]. WNCS over MANET is a comparatively new research area and is still thriving. Designing such a system brings new challenges to the researchers such as maintaining acceptable packet delay and packet drop using existing wireless technologies. The main focus of this paper is to identify and analyse the impact of network data rate, node mobility and ad hoc routing algorithms on the delay, drop and delay jitter of packets. Much of the research works on wireless networks is based on simulations as it is difficult and costly to launch real world experiments with mobile nodes [5]. Many simulation works assume simplified wireless communication that might produce impractical results. The major contribution of this paper is the implementation of a realistic wireless communication model using OPNET simulation to investigate the system performance of the distributed WNCS over MANET. This paper is organised as follows. Section II points to some previous work in the WNCS area, section III explains the simulation models, section IV presents the results that are analysed in section V. Finally, section VI draws some conclusions.

\section{PREVIOUS WORK}

Research papers such as [6], [7], [8], [9], [10], [11] etc. present brief survey and discussion of design issues for NCS. Most of the existing researches on WNCS revolve around two-node point to point wireless networks without intermediate nodes. The investigation of WNCS performance based on $802.11 \mathrm{~B}$ protocol for the inverted pendulum and tracking problem can be found in [12], [13]. TrueTime [14], [15] is a Matlab-Simulink based toolbox that allows performance evaluation of multitasking real time kernel executing various tasks with network support. TrueTime release 1.3 includes support for both wired and wireless (IEEE $802.11 \mathrm{~b} / \mathrm{g}$ and IEEE 802.15.4) network protocols. However, the wireless network block has limited support for MANET. Paper [16] discusses simulation of WNCS using TrueTime. A co-simulation of control and network, implemented in Matlab-Simulink, is presented in [3]. The authors investigated WNCS performance for various data rate, traffic, load etc. A general profile of wireless fieldbus has been introduced in [17]. The physical and data link layers of the proposed profile have been taken from existing wireless local area network and personal area network whereas the application layer is derived from wired fieldbus to provide better reliability. An overview of implementation of wireless networks in industrial applications can be found in [18]. The simulation of control mechanism over MANET for a simple first order system (water level control) has been discussed in [19]. Simple Simulink models for various design issues for WNCS such as packet delay, drop etc. are presented in [20].

\section{Simulation Model}

\section{A. Plant/controller model}

Soft real time systems e.g., temperature control, suffer only from reduced performance due to delays and remain stable as long as the delay magnitude is not excessively large. On the other hand, open loop unstable plants, e.g., inverted pendulum on a cart, are more difficult to control. Such systems are hard real time systems and must execute with a certain deadline. The effect of delay on the performance varies from one system to another [3]. To evaluate the overall system performance, both hard real time and soft real time plants are taken into account in this paper. Plants and controllers are implemented in OPNET using PROTO-C language.

1) Inverted pendulum control

The transfer function of the inverted pendulum system is given in (1) where the mass of the pendulum, $M=2 \mathrm{Kg}$, 
length of the pendulum, $L=0.5 \mathrm{~m}$, friction coefficient, $F=0.5 \mathrm{Nms} / \mathrm{rad}$ and gravitational acceleration, $g=10 \mathrm{~m} / \mathrm{s}^{2}$. It is clearly seen that the system is unstable without a proper controller. The adopted PID controller uses the proportional constant, $K_{P p}=24$, the integral constant $K_{P i}=5$ and the derivative constant, $K_{P d}=10$.

$$
\frac{Y(s)}{U(s)}=\frac{2}{s^{2}+s-20}
$$

\section{2) Temperature control}

The temperature control system has been taken from [3] and its transfer function is given in (2). The PI controller has proportional constant $K_{T p}=0.47$ and integral constant $K_{T i}=1.1$.

$$
\frac{Y(s)}{U(s)}=\frac{24.8}{s^{2}+10.2 s+20} \ldots
$$

3) Water level control

The water level model [21] is a first order system and its transfer function is given in (3). A PI controller with proportional constant $K_{W p}=10$ and integral constant $K_{W i}=5$ has been implemented for this plant.

$$
\frac{Y(s)}{U(s)}=\frac{15.8144}{63.2456 s+1}
$$

\section{B. Task invocation model}

The WNCS task invocation model applies clock driven sampling. Control/actuation tasks are invoked when an event occurs, for instance, when it receives an information packet from another node through the network [10], [22], [23]. The mechanism is explained in Figure 1. Once the controller receives a state packet, it determines the plant type and invokes appropriate control task. Upon receiving state packets from the inverted pendulum, temperature and water level plants, the controller executes the inverted pendulum control task followed by temperature control task followed by water level control task. Plants apply the control as soon as they receive a control packet. Clock driven sensing and event driven control-actuation approach has several advantages; it does not require plantcontroller synchronisation and supports multi-rate sampling [12].

Figure 2 depicts the scenarios that are considered in this paper. Figure 2A and Figure 2B show the direct control and control over MANET for a single plant, respectively. Figure 2C shows the mechanism of sharing the MANET among the plants for the distributed control. In the result section, performances of scenarios $\mathrm{B}$ and $\mathrm{C}$ have been compared with the scenario A.

\section{Network model}

One of the aims of this paper is to implement a realistic wireless signal propagation model for WNCS over MANET. A comparison between computer simulation and real world wireless network experiments can be found in [5], [24], [25]. Three different radio signal propagation models have been investigated in [5] as shown in Figure 3. The best model involves two components: path loss exponent and fading. The no variations model is the tworay-ground reflection model that uses only the path loss

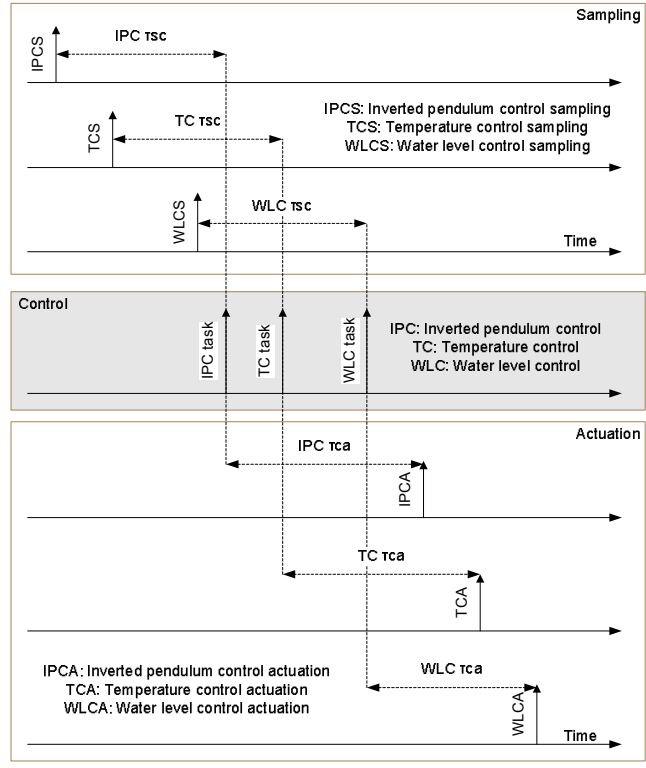

Figure 1: Task invocation model for sampling, control and actuation tasks.
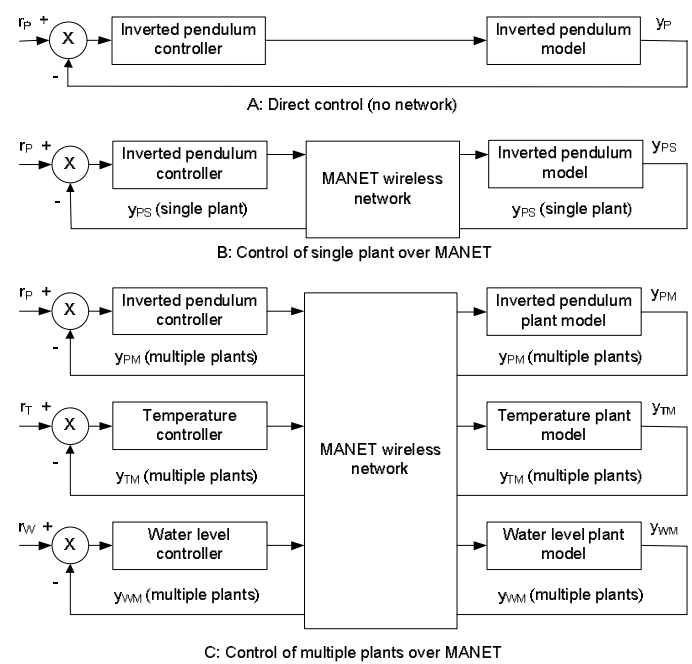

Figure 2: Scenarios for comparing system performance.

component. Finally, the perfect channel represents the ideal propagation model. The comparison, shown in Figure 3, revealed that the best model exhibits the closest behaviour to the real world experiment [5].

The radio propagation model used in this paper considers both path loss and fading (best model) to achieve very realistic signal propagation. The model is expressed in (4) where $P_{r}$ is the received wireless signal power, $\beta$ is path loss exponent, $d$ is the distance between the transmitter and the receiver, $d_{0}$ is the reference distance and $X_{d B}$ is a Gaussian random variable with zero mean and standard deviation $\sigma_{d B}$. Here $\sigma_{d B}$ is called fading deviation that can be obtained by measurement. This model extends the perfect channel or ideal circular model to a sensible statistic model in which nodes communicate probabilistically at the edge of the communication range [26].

$$
\left[\frac{P_{r}(d)}{P_{r}\left(d_{0}\right)}\right]_{d B}=-10 \beta \log \left(\frac{d}{d_{0}}\right)+X_{d B}
$$




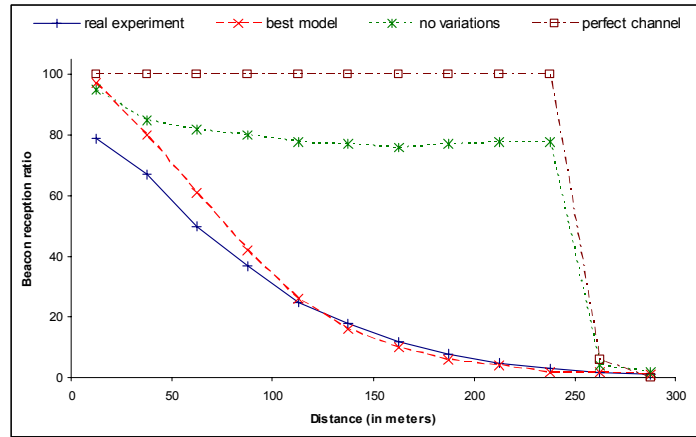

Figure 3: Comparison of three simulation models with real world experiment [5].

The simulation model implements IEEE $802.11 \mathrm{~b}$ technology that can support up to $11 \mathrm{Mbps}$ data rate. A square open field of size $222 \mathrm{~m}$ by $429 \mathrm{~m}$ with 40 MANET nodes equipped with Lucent Orinoco wireless network cards are taken into consideration [24]. To reflect the open field environment, path loss exponent, $\beta=2.8$ and $X_{d B}$ $=6 \mathrm{~d} B$ fading effect have been implemented in the OPNET simulation as suggested in [5], [24], [25].

\section{Node mobility model}

For movement, random way-point model has been implemented. In this model, nodes move from one point to another random point at a constant speed chosen from a specified range. It then waits at the new point for some time and then another random destination point is chosen. This movement model provides continuous node movement so that MANET routing algorithms can be evaluated [24], [25]. In this simulation, node speeds are chosen uniformly between 1 and $10 \mathrm{~m} / \mathrm{s}$. Nodes wait for 60 s before moving to a new destination point [24].

\section{E. Assumptions}

State and control information can be placed in a single packet. Total control loop delay should not exceed the sampling period and no delay compensation mechanism is implemented.

\section{REsults}

First the DSR routing algorithm [27] is used to investigate the performance for various sampling periods, network data rates and node movements. Then the AODV routing [28] is considered and compared to the DSR algorithm.

\section{A. Effect of sampling period}

The controller with three control algorithms (one for pendulum, one for temperature and one for water tank) has been placed at the centre of the field whereas the plants are located at the corners illustrated in Figure 4. To explore the impact of sampling period, the lowest data rate of $1 \mathrm{Mbps}$ has been implemented without any node movement under the DSR routing protocol. A sinusoidal wave with amplitude $1^{\circ}(0.0175$ radian $)$ has been taken as the reference signal. The largest acceptable sampling periods are given in Table I. For the rest of the paper, these sampling periods are considered for the investigation.

\section{B. Effect of data rate}

Lucent Orinoco wireless network cards support 1, 2, 5.5 and $11 \mathrm{Mbps}$ data rates with $-94,-91,-87$ and $-82 \mathrm{dBm}$ receiver sensitivities, respectively. In other words, as the data rate increases, the receiver needs higher signal power to receive packets properly thus reducing successful transmission range. Table II summarises the plant tracking performance for different data transmission rates under the DSR routing strategy.

TABLE I. MAXIMUM ACCEPTABle SAMPLING Periods

\begin{tabular}{|c|c|c|c|c|c|}
\hline & \multicolumn{3}{|c|}{ Reference signal, $r$} & \multicolumn{2}{|c|}{$\begin{array}{c}\text { Max sampling } \\
\text { period }\end{array}$} \\
\hline $\begin{array}{l}\text { Inverted } \\
\text { control }\end{array}$ & \multicolumn{3}{|c|}{$\mathrm{r}_{\mathrm{P}}=0.0175 * \sin (\mathrm{t})$} & \multicolumn{2}{|c|}{$0.02 \mathrm{~s}$} \\
\hline Temperature control & \multicolumn{3}{|c|}{$\mathrm{r}_{\mathrm{T}}=0.0175^{*} \sin (\mathrm{t})$} & \multicolumn{2}{|l|}{$0.2 \mathrm{~s}$} \\
\hline Water level control & \multicolumn{3}{|c|}{$\mathrm{r}_{\mathrm{W}}=1+0.0175 * \sin (\mathrm{t})$} & \multicolumn{2}{|l|}{$0.2 \mathrm{~s}$} \\
\hline \multicolumn{6}{|c|}{$\begin{array}{l}\text { TABLE II. DATA RATE AND PLANT TRACKING PERFORMANCE } \\
\text { (STATIONARY NODES, DSR ROUTING) }\end{array}$} \\
\hline & $\begin{array}{c}1 \\
\text { Mbps }\end{array}$ & $\begin{array}{c}2 \\
\text { Mbps }\end{array}$ & & $\begin{array}{l}.5 \\
\text { bps }\end{array}$ & $\begin{array}{c}11 \\
\text { Mbps }\end{array}$ \\
\hline $\begin{array}{l}\text { Distributed inverted } \\
\text { pendulum control }\end{array}$ & $\square$ & $\square$ & 区 & & \\
\hline $\begin{array}{l}\text { Distributed temperature } \\
\text { control }\end{array}$ & $\nabla$ & $\nabla$ & 区 & & $\nabla$ \\
\hline $\begin{array}{l}\text { Distributed water level } \\
\text { control }\end{array}$ & $\nabla$ & $\square$ & $\sqrt{v}$ & & $\nabla$ \\
\hline
\end{tabular}

Figure 4 shows the paths taken by the packets at data rate of $1 \mathrm{Mbps}$ (receiver sensitivity of $-94 \mathrm{dBm}$ ). As the receiver can receive low power signals properly, packets can reach the controller or plants directly (inverted pendulum) or through maximum 2 intermediate nodes. Therefore, packet delay and drop probability exhibit very low values. Figure 5 shows the paths under $11 \mathrm{Mbps}$ data rate. It is noted that packets experience at least two intermediate nodes before reaching its destination.

As data rate increases, transmission range becomes smaller and packets need to travel via more intermediate nodes to reach the destination. Therefore, the overall packet delay increases. As an example, packet delays and drops for the inverted pendulum control system are shown in Figure 6. State or control packets that experience delays more than $50 \%$ of the sampling period $(0.02 \mathrm{~s})$ are treated as obsolete and are dropped by the system. For $11 \mathrm{Mbps}$ data rate, packets experience significant delay jitter (delay standard deviation 0.0046s) and drops (approximately 22.77\%) making the plant unstable.

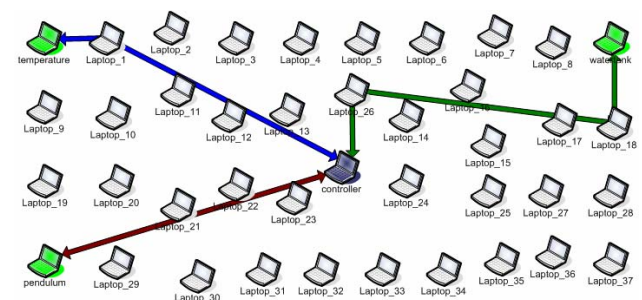

Figure 4: Packet routes for data rate of $1 \mathrm{Mbps}$ (DSR routing).

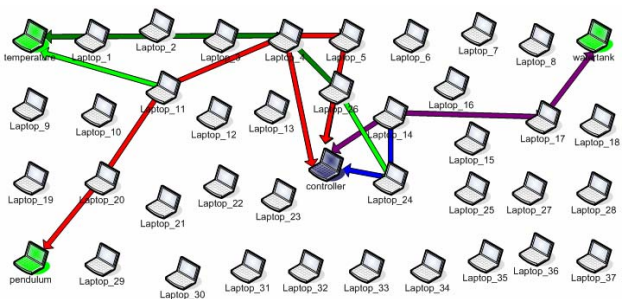

Figure 5: Packet routes for data rate of $11 \mathrm{Mbps}$ (DSR routing). 


\section{Effect of node movement}

This section explores the impact of random way-point node movement for different data rates under the DSR routing policy. Table III summarises the plant tracking performance under node movement for different data transmission rates. It is noted from Table III that the tracking performance window becomes narrower than Table II when movement is introduced. For stationary nodes, distributed control can be supported at data rates of 1, 2 and 5.5 Mbps for all plants (Table II). On the other hand, distributed control is supported for only data rates of 1 and 2 Mbps for mobile nodes (Table III).

TABLE III. DATA RATE AND PlANT TRACKING PERFORMANCE (NODE MOVEMENT, DSR ROUTING)

\begin{tabular}{|l|l|l|l|l|}
\hline & \multicolumn{1}{|c|}{$\begin{array}{c}\mathbf{2} \\
\text { Mbps }\end{array}$} & $\begin{array}{c}\mathbf{2} \\
\text { Mbps }\end{array}$ & $\begin{array}{c}\mathbf{5 . 5} \\
\text { Mbps }\end{array}$ & $\begin{array}{c}\mathbf{1 1} \\
\text { Mbps }\end{array}$ \\
\hline $\begin{array}{l}\text { Distributed inverted } \\
\text { pendulum control }\end{array}$ & $\square$ & $\square$ & & \\
\hline $\begin{array}{l}\text { Distributed temperature } \\
\text { control }\end{array}$ & $\square$ & $\square$ & & \\
\hline $\begin{array}{l}\text { Distributed water level } \\
\text { control }\end{array}$ & $\square$ & $\square$ & $\square$ & \\
\hline
\end{tabular}

Figure 7 shows the paths taken by packets for data rate of 1 Mbps under the DSR routing with node movements. It is noted that the plant and the controller can communicate directly (temperature control) or through maximum one intermediate node. Figure 8 shows the routes that are attempted by the packets before arriving at the destination under the data rate of $11 \mathrm{Mbps}$. Longer routes cause longer delays and make packets obsolete.

As node movement is introduced, more routes are taken by packets to reach destination. Route re-establishment produces longer delays, higher delay variation and drop probability. This can be easily identified from Figure 9 that shows the comparison of packet delays and drops between stationary nodes and mobile nodes for the inverted pendulum control system. Some packets are dropped (4.95\%) by the system for the data rate of $2 \mathrm{Mbps}$ under node movements.

The comparison of the tracking performance of the inverted pendulum control over 2 Mbps MANET with mobile nodes is depicted in Figure 10. $E P S=y_{P S}-y_{P}$ (Figure 2B) and $E P M=y_{P M}-y_{P}$ (Figure 2C) represent the inverted pendulum output errors for single pendulum and multiple plant distributed control, respectively, when compared to the output under direct control (Figure 2A). Distributed control exhibits higher error than the single pendulum control as the MANET needs to carry traffic for the

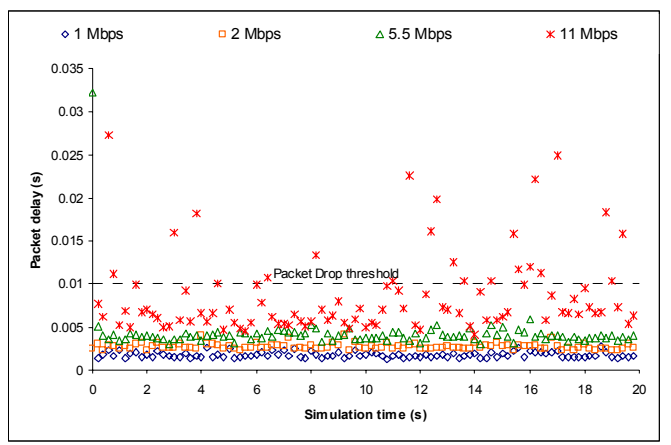

Figure 6: Packet delay for inverted pendulum (DSR routing).

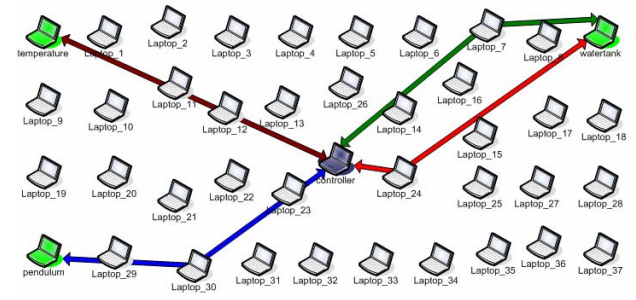

Figure 7: Packet routes for data rate of $1 \mathrm{Mbps}$ with node movement (DSR routing).

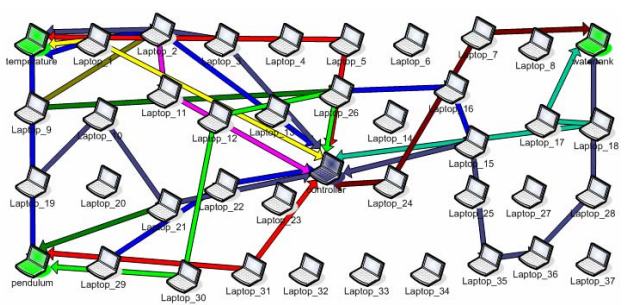

Figure 8: Packet routes for data rate of $11 \mathrm{Mbps}$ with node movement (DSR routing).

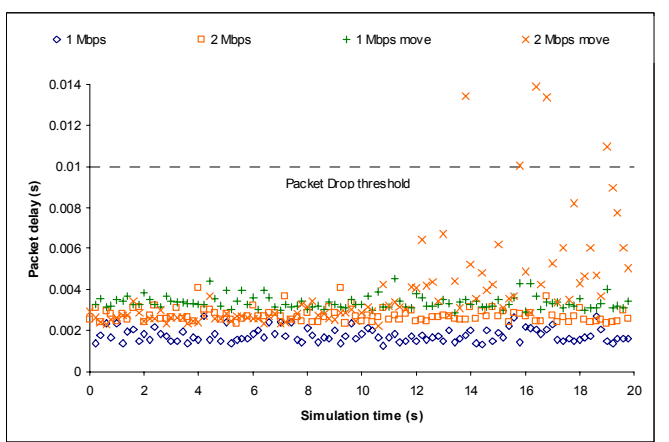

Figure 9: Comparison of packet delays between stationary nodes and mobile nodes (DSR routing).

temperature and the water level plants as well. Figure 11 shows the error comparison for the temperature plant over 2 Mbps MANET with mobile nodes where $E T S=y_{T S}-y_{T}$ and $E T M=y_{T M}-y_{T}$. Distributed control and single temperature control errors exhibit the same performance and are completely overlapping. For slow systems the distributed control and traffic from other plants is not as critical as the fast systems. The water level plant errors for single plant and distributed control are shown in Figure 12 where $E W S=y_{W S^{-}} y_{W}$ and $E W M=y_{W M^{-}} y_{W}$. Again, single plant and distributed control generate the same performance without any degradation as it is a slow or soft real time system.

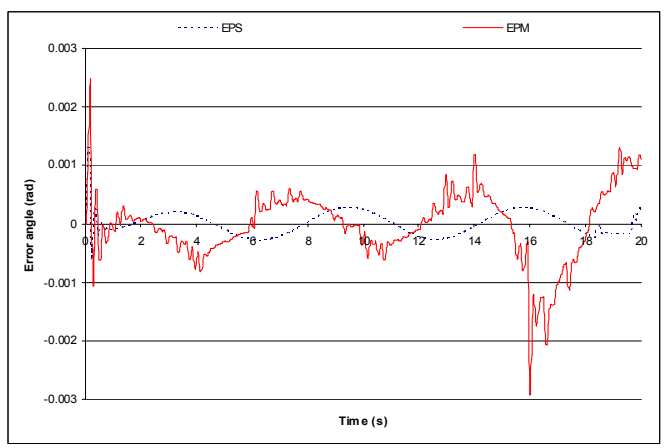

Figure 10: Comparison of the inverted pendulum angle error for single inverted pendulum and distributed control (three plants) over $2 \mathrm{Mbps}$ MANET with mobile nodes 


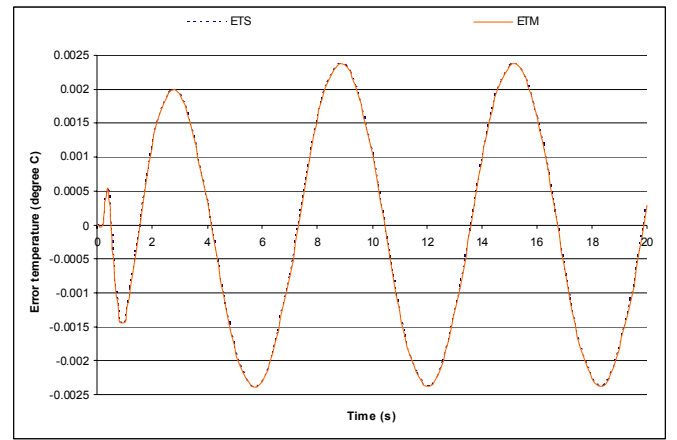

Figure 11: Comparison of the temperature plant output error for single temperature plant and distributed control (three plants) over $2 \mathrm{Mbps}$ MANET with mobile nodes

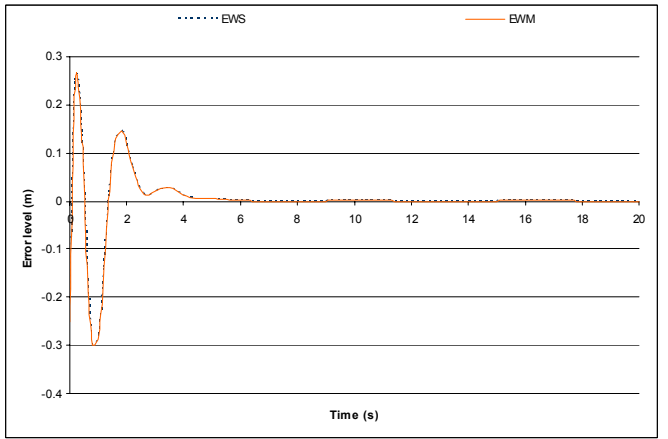

Figure 12: Comparison of the water level plant output error for single water level plant and distributed control (three plants) over $2 \mathrm{Mbps}$ MANET with mobile nodes

\section{Effect of routing algorithm}

Table IV and V summarise the plant tracking performance for data rates under the AODV routing protocol. It should be noted that as movement is introduced, temperature and water level control systems exhibit better performance. It might be the case that node movements allow better route establishment that support the delay and drop requirement of the plants. However, the inverted pendulum system supports only 1 Mbps data rate under node movement.

TABLE IV. DATA RATE AND PlANT TRACKING PERFORMANCE (STATIONARY NODES, AODV ROUTING)

\begin{tabular}{|l|l|l|l|l|}
\hline & \multicolumn{1}{|c|}{$\begin{array}{c}\mathbf{2} \\
\text { Mbps }\end{array}$} & $\begin{array}{c}\mathbf{5 . 5} \\
\text { Mbps }\end{array}$ & $\begin{array}{c}\mathbf{1 1} \\
\text { Mbps }\end{array}$ & Mbps \\
\hline $\begin{array}{l}\text { Distributed inverted } \\
\text { pendulum control }\end{array}$ & $\square$ & $\square$ & & \\
\hline $\begin{array}{l}\text { Distributed temperature } \\
\text { control }\end{array}$ & $\square$ & & & \\
\hline $\begin{array}{l}\text { Distributed water level } \\
\text { control }\end{array}$ & $\nabla$ & $\square$ & & \\
\hline
\end{tabular}

TABLE V. DATA RATE AND Plant TRACKIng PERFormance (NODE MOVEMENT, AODV ROUTING)

\begin{tabular}{|l|l|l|l|l|}
\hline & \multicolumn{1}{|c|}{$\begin{array}{c}\text { 2 } \\
\text { Mbps }\end{array}$} & $\begin{array}{c}\mathbf{2} \\
\text { Mbps }\end{array}$ & $\begin{array}{c}\mathbf{5 . 5} \\
\text { Mbps }\end{array}$ & $\begin{array}{c}\mathbf{1 1} \\
\text { Mbps }\end{array}$ \\
\hline $\begin{array}{l}\text { Distributed inverted } \\
\text { pendulum control }\end{array}$ & $\square$ & & & \\
\hline $\begin{array}{l}\text { Distributed temperature } \\
\text { control }\end{array}$ & $\square$ & $\square$ & & \\
\hline $\begin{array}{l}\text { Distributed water level } \\
\text { control }\end{array}$ & $\nabla$ & $\nabla$ & $\square$ & \\
\hline
\end{tabular}

Figure 13 depicts the packet delay and drop for the inverted pendulum control system under AODV routing. It is noted from the figure that some of the packet delays are larger than $0.01 \mathrm{~s}$ for 5.5 and $11 \mathrm{Mbps}$ data rates and these obsolete packets are dropped by the system. On the other hand, Figure 14 shows the comparison between stationary nodes and mobile nodes under the AODV routing protocol. As packets need to travel no or at best one intermediate node at $1 \mathrm{Mbps}$ data rate, the variation between stationary nodes and mobile nodes is not that significant.

\section{ANALYSIS}

Table VI shows the successful transmission ranges for the implemented wireless signal propagation model obtained from a separate OPNET simulation. The plants were located more than $205 \mathrm{~m}$ away from the controller. For 1 Mbps data rate (225m range), communication can be made directly or via one intermediate node. For a transmission range of $175 \mathrm{~m}$ at least one intermediate node is required to maintain the communication. As the range becomes even smaller more intermediate nodes are necessary to establish routes and hence the packet delay and drop probability increases.

TABLE VI. TRANSMISSION RANGE FOR DIFFERENT DATA RATES (RECEIVER SENSITIVITY)

\begin{tabular}{|l|c|c|c|c|}
\hline & $\begin{array}{c}\mathbf{1} \\
\text { Mbps }\end{array}$ & $\begin{array}{c}\mathbf{2} \\
\text { Mbps }\end{array}$ & $\begin{array}{c}\mathbf{5 . 5} \\
\text { Mbps }\end{array}$ & $\begin{array}{c}\mathbf{1 1} \\
\text { Mbps }\end{array}$ \\
\hline Transmission range & $225 \mathrm{~m}$ & $175 \mathrm{~m}$ & $125 \mathrm{~m}$ & $75 \mathrm{~m}$ \\
\hline
\end{tabular}

\section{CONCLUSIONS}

The main focus of this paper is to implement a realistic wireless signal propagation model to investigate the performance of distributed WNCS over MANET. It was found that the main challenges of such a system are to maintain acceptable packet delay and packet drops over the MANET. Increasing data rate makes transmission range smaller. Therefore, packets need to travel via more intermediate nodes to reach the destinations. This increases packet delay and drop probability. Furthermore,

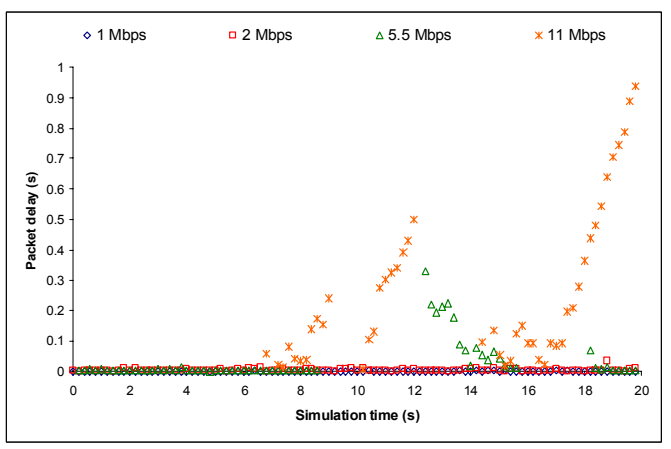

Figure 13: Packet delay for inverted pendulum (AODV routing).

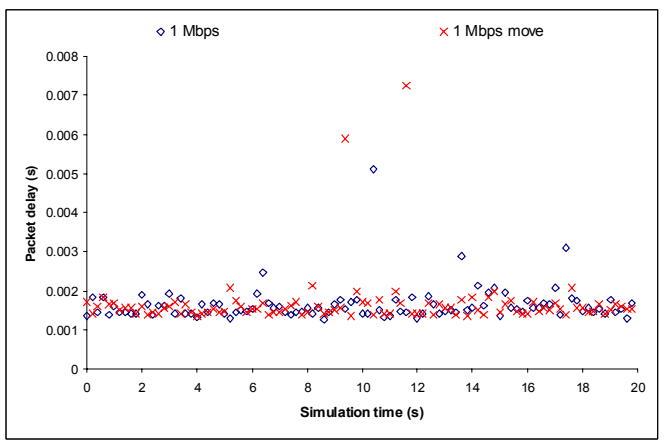

Figure 14: Comparison of packet delays between stationary nodes and mobile nodes (AODV routing). 
node movement makes the system tracking region even narrower. In case of node movements, the MANET needs to establish new routes that cause higher packet delay and drop. However, the DSR routing strategy exhibited better performance than the AODV routing strategy for both stationary nodes and mobile nodes.

Acknowledgements: The authors would like to thank the EPSRC research council (research grant EP/E025250/1) for the support of this research.

\section{REFERENCE}

[1] R. M. Murray, K. J. Astrom, S. P. Boyd, R. W. Brockett, and G. Stein, "Future Directions in Control in an information-rich world," IEEE Control Systems Magazine, vol. 23, no. 2, pp. 20-33, Apr. 2003.

[2] S. Mastellone and C. T. Abdallah, "Networked Control Systems and Communication Networks: Integrated Model and Stability Analysis," presented at the 16th IFAC World Congress, Prague, Czech Republic, Jul. 2005.

[3] J. Colandairaj, G. W. Irwin, and W. G. Scanlon, "Analysis and CoSimulation of an IEEE 802.11B Wireless Networked Control System," presented at the 16th IFAC World Congress, Prague, Czech Republic, Jul. 2005.

[4] E. M. Royer and C. K. Toh, "A Review of Current Routing Protocols for Ad Hoc Mobile Wireless Networks," IEEE Personal Communications, vol. 6, no. 2, pp. 46-55, 1999.

[5] D. Kotz, C. Newport, R. S. Gray, J. Liu, Y. Yuan, and C. Elliott, "Experimental Evaluation of Wireless Simulation Assumptions," presented at the ACM/IEEE International Symposium on Modelling, Analysis and Simulation of Wireless and Mobile Systems (MSWiM), pp. 78-82, Oct. 2004.

[6] T. C. Yang, "Networked Control System: A Brief Survey," IEE Proceedings - Control Theory and Applications, vol. 153, no. 4, pp. 403-412, Jul. 2006.

[7] T. C. Yang, H. Yu, M. R. Fei, and L. X. Li, "Networked control systems: a historical review and current research topics," the Journal of the Institute of Measurement and Control, vol. 38, no. 1, pp. 12-16, Feb. 2005.

[8] H. Yu, C. Harding, and M. S. Hasan, "Overview of Networks and Control," presented at the International Conference on Instrumentation, Control and Information Technology, Japan, pp. 205-210, Aug. 2005.

[9] P. Albertos, A. Crespo, M. Valles, and I. Ripoll, "Embedded Control Systems: Some Issues and Solutions," presented at the 16th IFAC World Congress, Prague, Czech Republic, Jul. 2005.

[10] K. E. Årzén and A. Cervin, "Control and Embedded Computing: Survey of Research Directions," presented at the 16th IFAC World Congress, Prague, Czech Republic, Jul. 2005.

[11] M. S. Branicky, V. Liberatore, and S. M. Phillips, "Networked Control System Co-Simulation for Co-Design," presented at the American Control Conference, Denver, Jun. 2003.

[12] N. J. Ploplys, "Wireless feedback control of mechanical systems," in Mechanical Engineering, MSc thesis, University of Illinois, Champaign, 2003, 2http://mrroboto.me.uiuc.edu/ARGWeb/theses/NicksThesis/Ploplys MS200 3.pdf
[13] N. J. Ploplys, P. A. Kawka, and A. G. Alleyne, "Closed-loop Control over Wireless Networks," IEEE Control Systems Magazine, vol. 24, no. 3, pp. 58-71, Jun. 2004.

[14] A. Cervin, D. Hanriksson, B. Lincoln, J. Eker, and K. E. ArZen, "How does control timing affect performance? Analysis and Simulation of Timing using Jitterbug and TrueTime," IEEE Control Systems Magazine, vol. 23, no. 3, pp. 16-30, Jun. 2003.

[15] A. Cervin, D. Henriksson, B. Lincoln, J. Eker, and K. E. Arzen, "Jitterbug and TrueTime: Analysis Tools for Real-Time Control Systems," presented at the 2nd Workshop on Real-Time Tools, Copenhagen, Denmark, Aug. 2002.

[16] M. Andersson, D. Henriksson, A. Cervin, and K. E. Arzen, "Simulation of Wireless Networked Control Systems," presented at the 44th IEEE Conference on Decision and Control and European Control Conference (ECC), pp. 476-481, Dec. 2005.

[17] F. D. Pellegrini, D. Miorandi, S. Vitturi, and A. Zanella, "On the Use of Wireless Networks at Low Level of Factory Automation Systems," IEEE Transactions on Industrial Informatics, vol. 2, no. 2, pp. 129-143, May. 2006.

[18] A. Willig, K. Matheus, and A. Wolisz, "Wireless Technology in Industrial Networks," Proceedings of the IEEE, vol. 93, no. 6, pp. 1130-1151, Jun. 2005.

[19] M. S. Hasan, C. Harding, H. Yu, and A. Griffiths, "Modeling delay and packet drop in Networked Control Systems using Network Simulator NS2," the International Journal of Automation and Computing (IJAC), vol. 2, no. 2, pp. 187-194, 2005.

[20] M. S. Hasan, H. Yu, A. Griffiths, and T. C. Yang, "Design Issues and Simulation models of Wireless Networked Control Systems," presented at the 12th Chinese Automation \& Computing Society Conference in the UK, Loughborough, pp. 123-128, Sep. 2006.

[21] K. Ogata, Modern Control Engineering, 4 ed. New Jersey, USA: Prentice Hall, 2002.

[22] J. Nilsson, "Real-time Control Systems with Delays," in Automatic Control, thesis, Lund Institute of Technology, Lund, Sweden, 1998, http://www.control.lth.se/documents/1998/nilj98dis.pdf.

[23] Y. Yang, Y. Wang, and S. H. Yang, "A Networked Control System with Stochastically Varying Transmission Delay and Uncertain Process Parameters," presented at the 16th IFAC World Congress, Prague, Czech Republic, Jul. 2005.

[24] J. Liu, Y. Yuan, D. M. Nicol, R. S. Gray, C. C. Newport, D. F. Kotz, and L. F. Perrone, "Simulation validation using direct execution of wireless ad-hoc routing protocols," presented at the 18th Workshop on Parallel and Distributed Simulation (PADS'04), May. 2004.

[25] C. Newport, "Simulating mobile ad hoc networks: a quantitative evaluation of common MANET simulation models," Dartmouth College Science Technical Report TR2004-504, Jun. 2004, http://cmc.cs.dartmouth.edu/cmc/papers/newport:thesis.pdf.

[26] K. Fall and K. Varadhan, "The Ns Manual (formerly ns Notes and Documentation), The VINT project," 2006, http://www.isi.edu/nsnam/ns/doc/ns doc.pdf, Mar 2006.

[27] D. B. Johnson, D. A. Maltz, and J. Broch, Ad Hoc Networking. Boston, USA: Addison-Wesley, 2001.

[28] C. E. Perkins and E. M. Royer, "Ad hoc On-Demand Distance Vector Routing," presented at the 2nd IEEE Workshop on Mobile Computing Systems and Applications (WMCSA 99), New Orleans, LA, pp. 90-100, Feb. 1999. 